\title{
New Approach for High Quality Cross Sectioning in Tablets using Broad Argon Ion Beam with $\mathrm{LN}_{2}$ Sample Cooling
}

\author{
Rintaro Kawano ${ }^{1}$, Yuji Hasebe ${ }^{1}$, Yuuki Yamaguchi ${ }^{1}$, Noriyoshi Manabe ${ }^{2}$, Natasha Erdman ${ }^{3}$ and \\ Shunsuke Asahina ${ }^{1}$ \\ 1. SM Business Unit, JEOL Ltd., Akishima, Tokyo, Japan \\ 2. Pharmaceutical Physical Chemistry, Tohoku Medical and Pharmaceutical University, Sendai, Japan \\ 3. JEOL USA, Inc. Peabody, MA, USA
}

Functions of pharmaceutical tablets such as sustained or enteric release in order to demonstrate well the effect of a medicine are controlled by their structure and components. Therefore, it is necessary to analyze both structures and components in order to understand their effects clearly. Nowadays, multiple methods are used to analyze these materials, for example X-ray Computed Tomography, Raman spectroscopy and Terahertz pulse imaging [1,2,3]. Moreover, analysis of medicine is also required to observe their structures directly on surface or cross section with Scanning Electron Microscopy (SEM) since their complex structures are becoming nano scale.

However, cross sections of tablets are generally very difficult to prepare due to fragility and softness. Preparation damage might cause sample deformation such as peel-off of the coating layers after razor blade cutting or mechanical polishing [4]. In this report, we applied broad argon ion beam process for high quality cross sectioning in pharmaceutical tablets. We also utilized liquid nitrogen $\left(\mathrm{LN}_{2}\right)$ during the ion milling, since some tablets contain substances with low-melting point such as starch and beeswax as thermal damage in the process might cause sample deformation.

Nifedipine tablet of generic drug which is used for treating of hypertension was processed by normal milling without cooling and with cooling at $-120^{\circ} \mathrm{C}$. These cross sections were carried out by using IB19530CP or IB-19520CCP (JEOL Ltd) respectively [5]. Milling conditions are acceleration voltage of 5 $\mathrm{kV}$ and milling time of $7 \mathrm{hr}$. The cross sections were coated with osmium. SEM (JSM-IT500HR, JEOL) images were captured at $5 \mathrm{kV}$. Energy dispersive X-ray spectrometer (EDS) spectra and elemental maps were taken with a JEOL JSM-7800FPRIME equipped with the Thermo Fisher Scientific dual windowless EDS system at $7 \mathrm{kV}$.

Figure 1 shows comparison between preparations at room and $\mathrm{LN}_{2}$ temperature. There are minimal voids with cooling process, while lack of cooling shows voids. Therefore, the cooling system during Ar ion milling is suitable for cross sectioning in pharmaceutical tablets. And we have tried also comparison with original and generic drugs to understand the structures with cooling system that is shown in Figure 2. As the result, the thickness and sizes of granules in pellets differ greatly between original and generic drugs. In addition, Talc $\left(\mathrm{Mg}_{3} \mathrm{Si}_{4} \mathrm{O}_{10}(\mathrm{OH})_{2}\right)$ as a lubricant was detected in coating layer of the generic drug, however it was not detected in the original drug that is shown in Figure 3.

We are able to analyze for pharmaceutical tablets with SEM that give us better understanding of the structure and the components of tablets even with nano scale after preparing high quality cross sections. This method can contribute to future drug design and research of medicinal effect based on correlation to dissolution property and weight of tablets. 


\section{Reference:}

[1] D. Taraini et al, Microscopy and Analysis 22 (2008), p. 13.

[2] A. Kuriyama et al, AAPS PharmSciTech 15 (2014), p. 375.

[3] L. Ho et al, Journal of Controlled release 127 (2008), p. 79.

[4] Y. Fu et al, Journal of Controlled release 109 (2005), p. 203.

[5] S. Asahina et al, APL Materials 2 (2014), p. 113317

(a)

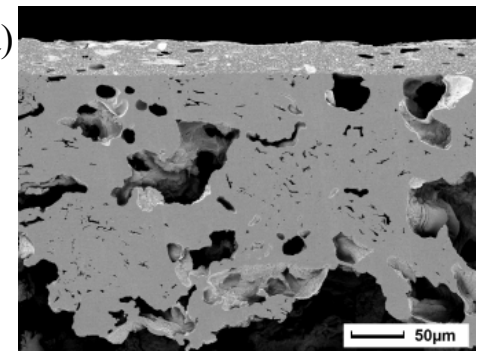

(b)

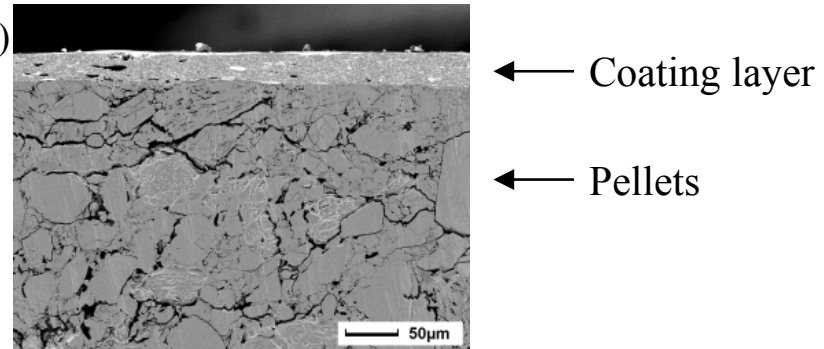

Figure 1. Cross section processed (a) at room temperature and (b) $-120{ }^{\circ} \mathrm{C}$.

(a)

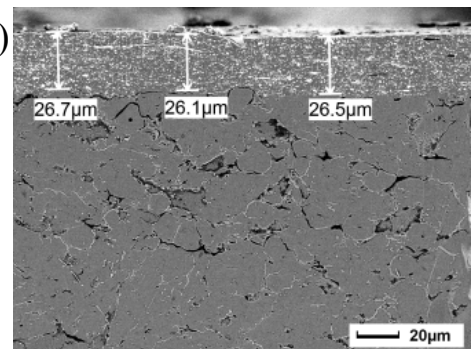

(b)

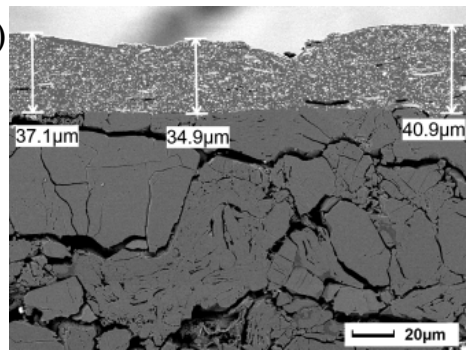

Figure 2. Cooled processing of (a) original drug and (b) generic drug.
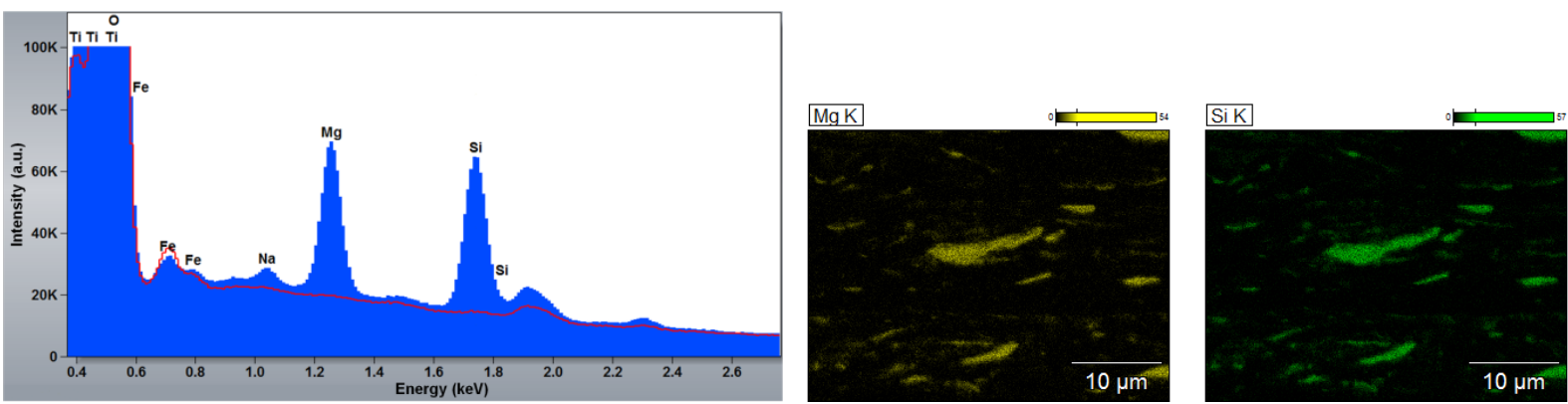

Figure 3. Spectrum comparison of tablets cross section. The spectrum filled with blue is that of generic and the red line is that of original. And elemental maps of the generic drug coating layer. 